\title{
Sistemas de informação como indicadores de qualidade na saúde. Uma revisão de níveis de abordagem
}

\author{
Sílvia Fernandes ${ }^{1}$, Eugénia Tareco² \\ sfernan@ualg.pt, gena_34@hotmail.com \\ ${ }^{1}$ CIEO- Centro de Investigação sobre Espaço e Organizações, Universidade do Algarve, Campus de \\ Gambelas, 8005-139 Faro, Portugal \\ ${ }^{2}$ Faculdade de Economia, Universidade do Algarve, Campus de Gambelas, 8005-139 Faro, Portugal
}

DOI: 10.17013/risti.19.32-45

\begin{abstract}
Resumo: A introdução de novas tecnologias nas diversas áreas de atividade cria repercussões a nível económico, social e organizacional. As organizações de saúde devem acompanhar os novos padrões de exigência para responder aos desafios atuais. Os sistemas de informação em saúde (SIS) têm como objetivo promover a qualidade e continuidade dos cuidados prestados. Nesse sentido, os principais objetivos deste trabalho são: 1) identificar vantagens e desvantagens dos SIS, em particular na enfermagem, para a qualidade do cuidado e 2) avaliar se tais sistemas podem ser indicadores de qualidade. Trata-se de um estudo de revisão da literatura, de artigos científicos relacionados com estes objetivos. A maioria dos artigos analisados refere que os sistemas de informação em enfermagem (SIE) são uma importante ferramenta de trabalho e avaliação de qualidade. Existe uma perspetiva crescente baseada em resultados e inteligência organizacional. Aqui reside um importante fator de desempenho, aspeto muito relevante na área da saúde.
\end{abstract}

Palavras-chave: Sistemas de informação de saúde, sistemas de informação de enfermagem, qualidade dos cuidados, indicadores de qualidade, desempenho.

\begin{abstract}
The introduction of new technologies in several areas of activity creates impact at the economic, social and organizational level. Health organizations must keep up with new demand patterns to respond to current challenges. Health information systems (HIS) aim to promote quality and continuity of healthcare. Thus, the main objectives of this work are: 1) to identify advantages and disadvantages of health information systems, particularly in nursing, for the quality of care and 2) assess whether such systems can be quality indicators. It uses a literature review, of scientific articles related to these goals. Most of these articles states that nursing information systems (NIS) are an important tool of work and quality assessment. There is a growing perspective of HIS oriented to results and organizational intelligence. Here lies an important factor of performance, which is very relevant in health.
\end{abstract}

Keywords: Health information systems, nursing information systems, quality of healthcare, quality indicators, performance. 


\section{Sistemas de Informação em Enfermagem}

Os SIS estão em permanente evolução, o que torna inevitável a necessidade de disponibilizar sistemas capazes de se configurarem para uma utilização da informação orientada à qualidade e aos resultados (Pinto, 2009). Em particular, um sistema de informação em enfermagem (SIE) é definido para documentar e apoiar os cuidados prestados ao utente/família, comunicar com outros departamentos hospitalares, recuperar dados dos pacientes e impedir o uso indevido da informação (Freixo \& Rocha, 2014; Pinto, 2009). Para Sousa (2005) os SIE servem de suporte legal dos cuidados de enfermagem, dão visibilidade aos contributos do processo de enfermagem, facilitam a gestão, promovem a avaliação e a continuidade do cuidado. Os SIE devem dar resposta às necessidades dos enfermeiros: incluir a classificação de enfermagem, reduzir a duplicidade de dados e aumentar a acessibilidade à informação. Os registos de enfermagem devem incorporar as necessidades de cuidados ao utente, as intervenções de enfermagem e seus resultados (Rocha \& Rocha, 2013).

A importância dos registos em enfermagem é evidente em assegurar a continuidade dos cuidados, por permitirem um acesso a todas as informações do paciente de modo seguro e confidencial (Lima et al., 2011). Os SIE permitem elaborar diagnósticos personalizados, documentar os procedimentos e melhorar a qualidade da assistência (Lima \& Melo, 2011). Os registos podem ser assim indicadores de qualidade dos cuidados. Através de uma auditoria aos registos eletrónicos de enfermagem é possível analisar os cuidados prestados, repercussões e custos envolvidos. A auditoria de enfermagem é um instrumento de controlo de qualidade do trabalho da equipa. Assim, os cuidados de enfermagem podem ser avaliados pelos registos eletrónicos (Setz \& D’Innocenzo, 2009; Hesbeen, 2001; Martins et al., 2008).

\subsection{Sistemas de Informação para a Qualidade dos Cuidados de Enfermagem}

A continuidade do cuidado é um dos aspetos que permite avaliar a qualidade dos serviços de saúde, o que comprova a importância que esta dimensão tem em toda a dinâmica dos cuidados (Mota et al., 2014). Os SIE desempenham um papel fulcral na avaliação da qualidade, pois permitem aceder em tempo útil aos registos recolhidos, processados e documentados pelos enfermeiros. Definir qualidade não é simples, ao avaliar a excelência de um produto ou serviço (Ascenção, 2010; Bastos \& Saraiva, 2009). O conceito de qualidade foi desenvolvido durante a era industrial e foi adaptado à saúde por Avedis Donabedian ${ }^{1}$. Na saúde, envolve as dimensões: estrutura (recursos materiais, equipamentos e humanos); processo (relação entre o paciente e o prestador); e resultados (produto final da assistência prestada). Qualidade em saúde pode ser definida como a prestação de cuidados de saúde acessíveis e equitativos, com um nível profissional ótimo que tenha em conta os recursos disponíveis e alcance a adesão e satisfação do utente (PNS, 2012-2016). Trata-se de uma exigência de todos os envolvidos nos cuidados de saúde; é hoje uma dimensão incontornável na prestação de cuidados.

\footnotetext{
${ }^{1}$ Médico armênio reconhecido como o precursor do método de avaliação de qualidade na saúde. Desenvolveu um quadro concetual para o entendimento da avaliação da qualidade em saúde a partir dos conceitos de estrutura, processo e resultado.
} 
Pode ser influenciada pela formação profissional, número de profissionais por turno e estruturas das organizações. A melhoria da qualidade do cuidado deve fazer parte do dia-a-dia de cada um na realização de suas tarefas (Ascenção, 2010). Por isso, a avaliação da qualidade deve englobar todos os elementos da equipa multidisciplinar envolvida.

Em particular, os cuidados de enfermagem têm como finalidade a contínua melhoria da prestação, bem como definir estratégias para alcançar tal melhoria com base no custobenefício. A qualidade deve ser vista como um processo contínuo, estando diretamente relacionada com a qualidade da informação disponível (Mota et al., 2014). Para fazer face a estas necessidades, existem várias ferramentas que contribuem para a melhoria contínua da qualidade exigida aos hospitais (Bastos \& Saraiva, 2009). A nível hospitalar, a certificação poderá ser requerida a nível departamental, isto é, cada um dos serviços hospitalares (imunologia, laboratório, pediatria, etc.) pode desenvolver um sistema individual de gestão da qualidade solicitando em seguida uma auditoria com vista a obter uma certificação $\mathrm{ISO}^{2}$. O facto de uma organização se encontrar em conformidade com a norma significa que possui uma abordagem sistemática para a gestão dos processos orientada a que os produtos ou serviços prestados respondam às expetativas dos clientes. Segundo a ordem dos enfermeiros, a implementação de SI na área de enfermagem deve respeitar a norma ISO18104. Esta norma facilita os conceitos de diagnóstico e ações de enfermagem de modo a favorecer a sua manipulação nos SI.

A aplicação de questionários sobre a qualidade é outra ferramenta que os hospitais podem utilizar para avaliar os cuidados de enfermagem através dos registos. A qualidade pode ser avaliada através de auditorias aos processos de enfermagem. A sua função não é somente identificar falhas ou problemas, mas também apontar sugestões e soluções que promovam a qualidade dos cuidados (Setz \& D’Innocenzo, 2009). O processo eletrónico do doente abrange todos os cuidados prestados e deve transparecer todas as intervenções. O cuidado de enfermagem é complexo, abrangente e desafiador e os enfermeiros estão expostos a situações clínicas difíceis (Sasso et al., 2013). O processo de enfermagem tem tido os SI como grande aliado através da redução do tempo de documentação e registo clínico, da discussão dos casos e busca contínua de evidências nos dados. O maior desafio é determinar que informação o enfermeiro necessita de registar para promover a continuidade e qualidade do cuidado, uma vez que o volume de informação cresce. Com os SIE, podem organizar e determinar quais os registos essenciais para a promoção do cuidado. O resumo mínimo de dados em enfermagem representa a primeira tentativa de padronizar os dados essenciais para a prática de enfermagem. Entende-se por resumo mínimo de dados o conjunto mínimo de elementos de informação, com definições e categorias uniformes específicos da enfermagem, que satisfaz as necessidades dos utilizadores (Barra \& Sasso, 2011; Ordem dos Enfermeiros, 2007).

Os SIE são ferramentas que permitem otimizar o fluxo de trabalho e a eficácia do cuidado através do aumento do conhecimento dos profissionais, melhor articulação e comunicação entre as instituições e diminuição do tempo de espera pelo utente (Azevedo, 2010). Contudo, ainda existem dificuldades na sua implementação tais como:

\footnotetext{
${ }^{2}$ International Organization for Standardization.
} 
pouca adesão dos enfermeiros (por défice de conhecimento das TI), não envolvimento dos enfermeiros na implementação dos SI e elevados custos dos mesmos.

\subsection{Sistema de Informação como Indicador de Qualidade dos Cuidados}

Tendo em conta que o maior grupo profissional da área da saúde são os enfermeiros, é assim o grupo que mais decisões toma e atos regista. Pela especificidade de suas funções, produzem mais informação clínica sobre os pacientes (Pinto, 2009). A prática diária da profissão de enfermagem encontra-se diretamente relacionada com a aquisição, análise e interpretação de informação com uma constante necessidade de documentar dados inerentes à prestação de cuidados (Pinto, 2009). A necessidade de maior acessibilidade para continuidade do cuidado entre diferentes contextos (hospital, centros de saúde) esteve na origem da construção de plataformas de informação de enfermagem (Azevedo \& Sousa, 2012). A introdução dos SIE constitui uma mais-valia na profissão de enfermagem, dando visibilidade aos contributos dos cuidados de enfermagem para os ganhos em saúde das populações.

Os registos são a base de toda a metodologia de trabalho em enfermagem, pelo que devem ser rigorosos e completos. Reforçam assim a autonomia e a responsabilidade profissional (Martins et al., 2008). O sistema de avaliação de desempenho da carreira de enfermagem indica que existe deficiente desempenho se há incumprimento do dever de registo sistematizado dos cuidados de enfermagem. A implementação dos SIE veio preencher uma lacuna existente na profissão. Perante estes factos, um suporte dos SIE direcionado para os resultados poderá constituir um indicador de qualidade do trabalho de enfermagem e de saúde. Indicador de qualidade é um instrumento que permite verificar se os objetivos propostos foram ou não alcançados (D’Innocenzo et al., 2006). Santos e Rennó (2013) referem que indicadores são medidas de desempenho, ou seja, instrumentos com foco no resultado esperado e no processo de obtenção de resultados na qualidade do serviço. A finalidade dos indicadores é analisar as condições do serviço e compará-las com os padrões estabelecidos, contribuindo para a busca de excelência. Um indicador é um dado que gera informações sobre assuntos que merecem atenção e que possibilita a mudança de comportamentos de modo a atingir os objetivos propostos. As administrações hospitalares devem adotar indicadores de qualidade para avaliar a qualidade dos cuidados aí prestados. Alguns desses indicadores são: rotatividade dos funcionários, absentismo, taxa de ocupação hospitalar, tempo médio de internamento, índice de satisfação do utente, entre outros.

O Serviço Nacional de Saúde Português é um dos melhores do mundo, de acordo com um estudo do Boston Consulting Group (BCG). Contudo, existem ineficiências de que o BCG faz recomendações, uma das quais passa pela alteração do modo de financiamento hospitalar através de modelos orientados a resultados (Simões, 2015). Os indicadores produzidos pela integração dos SI têm aqui um papel fundamental. A literatura evidencia que a maioria dos indicadores utilizados para avaliar os cuidados de enfermagem está associada à estrutura e ao processo (Caldana et al., 2013), ou seja, ao que se relaciona com os cuidados diretos ao utente (Almeida \& Fontes, 2013). Torna-se importante que os enfermeiros aprofundem seus conhecimentos sobre os SIE (Pinto, 2009). As revisões sistemáticas de literatura na área podem ser o elo de ligação entre a pesquisa sobre SI e 
a prática clínica, no sentido de conhecer a perspetiva dos mesmos como indicadores de qualidade dos cuidados.

\section{Referencial Metodológico}

Com base nas características da problemática referida, os principais objetivos do presente trabalho são: 1) identificar vantagens e desvantagens dos SIS, em particular na enfermagem, para a qualidade dos cuidados e 2) avaliar se tais sistemas podem ser indicadores de qualidade. A investigação realizada enquadra-se no domínio da metodologia de revisão sistemática da literatura. Existem duas categorias de revisão: as revisões narrativas e as revisões sistemáticas, tendo características e objetivos diferentes. As revisões narrativas são apropriadas para descrever a história ou desenvolvimento de um problema, bem como para discutir o assunto do ponto de vista teórico. Não informam a metodologia de busca, nem critérios utilizados para a seleção dos artigos. A revisão sistemática é uma atividade fundamental para a prática baseada na evidência, pois condensa o conhecimento fracionado num único estudo, permitindo ao investigador uma cobertura mais ampla do que obteria com a realização de um estudo clínico (Pocinho, 2008).

O método de revisão sistemática da literatura é uma metodologia rigorosa, proposta para identificar os estudos sobre um tema em questão, aplicando critérios explícitos de busca (Sampaio\&Mancini, 2007; Gomes \& Caminha, 2014). Oinvestigador utiliza uma metodologia clara e bem definida, planeada para responder a uma pergunta específica usando métodos para identificar, selecionar, avaliar criticamente e analisar os dados. Pode estar ancorada em pesquisas qualitativas ou quantitativas, dependendo do objetivo de estudo. Uma revisão sistemática deve estar ancorada nos princípios metodológicos de uma revisão da literatura com base nas orientações da Colaboração Cochrane ${ }^{3}$. Esta estabelece sete passos para a realização de uma revisão sistemática: 1) formulação da pergunta, 2) localização e seleção dos estudos, 3) avaliação crítica dos estudos, 4) recolha de dados, 5) análise e apresentação dos dados, 6) interpretação e 7) atualização da revisão (Pocinho, 2008):

1. Formulação da pergunta: o primeiro passo a ser dado no início de qualquer estudo é estabelecer o que se deseja pesquisar. Questões mal formuladas podem conduzir a decisões obscuras sobre o que incluir na revisão.

2. Localização e seleção dos estudos em bases de dados: esta etapa realiza-se em bases de dados eletrónicas, revistas, livros. É necessário selecionar as bases eletrónicas adequadas à temática da saúde.

3. Avaliação crítica dos estudos: durante a seleção dos estudos, a avaliação dos títulos e dos resumos identificados na busca inicial deve obedecer aos critérios de inclusão e exclusão definidos no protocolo de pesquisa. Os critérios de inclusão e exclusão são definidos com base na pergunta a investigar.

4. Recolha de dados: etapa na qual é recolhido um conjunto de informações sobre cada estudo selecionado; feita depois da identificação e seleção dos estudos. A

\footnotetext{
${ }^{3}$ Organização criada para responder à necessidade de organizar de forma sistemática os resultados de investigação em saúde, de modo a facilitar a tomada de decisões médicas e ajudar a compreender em que campo é necessária mais investigação.
} 
sistematização é importante para evitar enviesamentos. A qualidade da revisão sistemática depende da validade dos estudos incluídos nela.

5. Análise e apresentação dos dados: com base nas semelhanças entre artigos, os dados são agrupados para a obtenção de conclusões. Cada um destes agrupamentos deve ser pré-estabelecido, evitando a tendenciosidade. Os artigos incluídos podem ser apresentados numa tabela que destaque suas características principais como: autores, ano de publicação, metodologia, variáveis dependentes e principais resultados.

6. Interpretação dos dados: a interpretação de resultados deve ser feita levando em conta a questão em estudo (estabelecida no primeiro passo).

7. Atualização da revisão: manter a revisão sistemática atualizada de acordo com novas pesquisas que surgem sobre o tema.

A secção seguinte aplica cada um dos passos anteriores de realização de uma revisão sistemática, sobre a questão e objetivos propostos, segundo as orientações da abordagem Cochrane. Também foram consideradas as orientações da abordagem PRISMA ${ }^{4}$ (Moher et al., 2009) que refere a importância dos termos de pesquisa, da sistematização dos critérios de inclusão/exclusão, da tabela de artigos selecionados e da frequência de expressões (Prisma checklist). Seguir as abordagens de referência permite melhorar a qualidade e rigor das revisões sistemáticas e meta-análises. É de realçar que a metaanálise é o método estatístico utilizado na revisão sistemática para integrar os resultados dos estudos incluídos (Castro, 2001).

\section{Investigação Realizada}

\subsection{Questão de Investigação}

A primeira decisão ao preparar uma revisão sistemática da literatura é determinar o seu enfoque. Muitas vezes os enfermeiros deparam-se com situações para as quais não possuem conhecimentos suficientes para a prestação de cuidados. Assim, a formulação de questões bem estruturadas permite diminuir incertezas relacionadas com a atividade. Esta etapa é muito importante visto que guia todo o processo metodológico. A questão que queremos explorar está relacionada com os seguintes aspetos complementares: 1) identificar vantagens e obstáculos aos SI para a qualidade dos cuidados de enfermagem, e 2) avaliar como os SI contribuem como indicador de qualidade dos cuidados prestados. A revisão sistemática será conduzida no sentido de discernir até que ponto se discute esta problemática e se abordam os SIE com base nos resultados, isto é, na sua capacidade de produzirem indicadores para a qualidade dos cuidados e serviços.

\subsection{Descritores}

Tendo em conta a questão atrás proposta, a pesquisa de publicações nas bases de dados foi realizada de acordo com os seguintes descritores: "sistemas de informação em saúde";

\footnotetext{
${ }^{4}$ Conjunto mínimo baseado em provas de itens para realizar revisões sistemáticas e metaanálises. Pode ser usado como base para realizar revisões sistemáticas de investigação sobre intervenções na saúde (estudos de diagnóstico, estudos de observação, etc.).
} 
"sistemas de informação em enfermagem"; "qualidade dos cuidados de enfermagem"; "indicadores de qualidade dos cuidados" (e sinónimos em inglês e espanhol). Por sua vez, o operador booleano 'AND' permite encontrar artigos que reúnam duas ou mais ideias. Isto significa que ambos os termos deverão surgir algures no artigo.

\subsection{Critérios de Inclusão e Exclusão}

O que distingue uma revisão sistemática de uma narrativa é a especificação de critérios, sendo importante a delimitação dos mesmos no início da revisão. Foram considerados os seguintes critérios de inclusão:

- Artigos publicados em português, espanhol ou inglês no período de 2009 a $2013^{5}$.

- Todas as categorias de artigo.

- Artigos com resumo e texto completos.

- Artigos contendo nos seus títulos ou resumos os descritores supra mencionados.

- Artigos dirigidos a profissionais de saúde.

Quanto aos critérios de exclusão, foram excluídos do estudo: os artigos dirigidos a outros profissionais (educadores de infância, etc.); artigos que fazem apenas uma breve descrição dos SI (definição, funcionalidade, software); e artigos que, após leitura do texto, não referem vantagens/desvantagens dos SI nem mencionam indicadores de qualidade em saúde.

\subsection{Pesquisa Eletrónica}

As revisões sistemáticas de literatura requerem uma pesquisa ampla e objetiva de modo a identificar o maior número de estudos. A identificação e seleção dos estudos respeitaram as recomendações estabelecidas pelas abordagens Cochrane e PRISMA (Moher et al., 2009). A recolha de dados decorreu entre Março e Agosto de 2013. A pesquisa foi efetuada nas plataformas SCIELO e EBSCO (bases de dados CINHAL e MEDLINE). A primeira plataforma foi acedida através do portal da Biblioteca Virtual de Saúde e a segunda através da Ordem dos Enfermeiros.

\section{Seleção de Artigos}

A seleção dos artigos baseia-se em critérios previamente estabelecidos, consistindo em duas fases: 1) leitura do título e resumo (abstract) dos artigos encontrados (nesta fase efetua-se uma pré-seleção de artigos potencialmente importantes para a revisão); e 2) leitura integral dos estudos selecionados. Decidir sobre os artigos que devem ser incluídos na revisão está refletido na sequência da figura 1.

Após a seleção final dos 18 artigos, foi efetuada uma nova leitura para a recolha das expressões ou palavras mais frequentes (tabela 1) tendo por base os objetivos do

\footnotetext{
${ }^{5}$ Este período tem a ver com o prazo de um projeto em que este trabalho se insere.
} 
2373 artigos obtidos

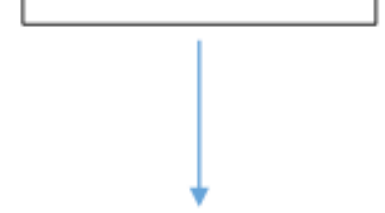

10 artigos excluidos por estarem

repetidos nas bases de dados

2363 artigos para seleçâo

73 artigos selecionados para leitura do resumo

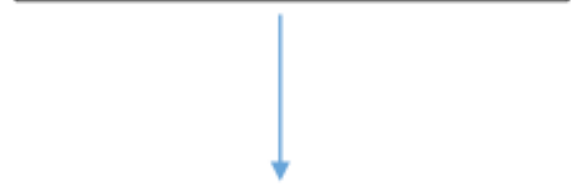

56 artigos para leitura integral do texto

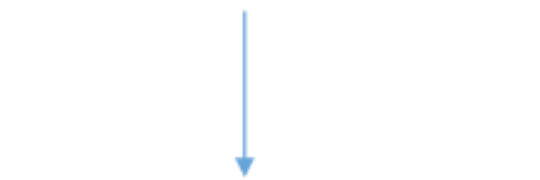

18 artigos selecionados para efetuar a revisão
17 artigos excluidos após a leitura do

resumo: fazem descrição dos SI em

termos técnicos (software)
38 artigos excluidos após leitura

integral: não referem desvantagens dos

SI; e não falam na importância da

qualidade dos cuidados

Figura 1 - Seleção dos Artigos Relevantes

presente trabalho. Os dados foram assim organizados com base em duas categorias, para depois se proceder à análise dos mesmos: sistemas de informação para a qualidade dos cuidados em enfermagem; e sistemas de informação como indicador de qualidade dos cuidados/serviços. 


\begin{tabular}{lll}
\hline Questão de investigação & Expressões ou palavras & Frequência \\
\hline \multirow{4}{*}{ objetivos dos SI em enfermagem } & rapidez de acesso & 7 \\
& disponibilidade & 3 \\
& facilidade de acesso & 5 \\
& clareza & 6 \\
\hline \multirow{4}{*}{ vantagens e desvantagens dos SIE } & otimização do espaço físico & 1 \\
& tomada de decisão & 7 \\
& comunicação entre profissionais & 5 \\
& poucos conhecimentos de TI & 4 \\
& sistema sujeito a falhas & 3 \\
qualidade dos cuidados de & sistema inoperante & 2 \\
enfermagem & avaliação dos cuidados de enfermagem & 10 \\
& qualidade dos registos & 6 \\
& podem ser auditados & 4 \\
& pouco tempo para o cuidado direto & 3 \\
& processos individualizados & 5 \\
\hline
\end{tabular}

Tabela 1 - Frequência e seleção de expressões/palavras

Posteriormente procedeu-se a uma leitura minuciosa de cada artigo e agruparam-se os mesmos por similaridade de conteúdo. Os documentos utilizados neste estudo são de caráter público e de livre acesso. A tabela 2 agrupa os aspetos realçados pela revisão e análise de conteúdo, separando-os por níveis: 1) gestão/operação; 2) avaliação/auditoria

'Nível' abordado

gestão/operação

(8 artigos)

\section{Aspetos focados (relacionados com a presente investigação)}

os SI disponibilizam de forma rápida e segura as informações necessárias aos profissionais de saúde. Todas as áreas que venham a trabalhar com a saúde podem usufruir dessas informações. São ferramentas que garantem constante atualização, fácil acesso e planeamento dos processos de enfermagem. Aumentam a eficiência dos serviços (informações em tempo útil). Um obstáculo é o financiamento para promover o desenvolvimento e implementação de SI. E ainda há algumas resistências à adesão e utilização de SI

os registos eletrónicos permitem agilizar dados de modo a fazer diagnósticos e avaliar as intervenções. Permitem ainda avaliar a produtividade e qualidade do cuidado prestado. Os registos eletrónicos promovem a continuidade do cuidado, pois os dados estão disponíveis em tempo real. A auditoria permite detetar problemas nos registos de enfermagem, por meio de relatórios de avaliação, orientando a equipa a melhorar registos

um sistema informatizado de indicadores de enfermagem permite armazenar dados pertinentes no processo de enfermagem $\mathrm{e}$ disponibiliza-los para avaliação de resultados. Daí os enfermeiros têm a responsabilidade de gerir o seu serviço por resultados. Os indicadores permitem avaliar, planear e organizar as atividades, fornecendo informação para a tomada de decisão

Tabela 2 - Níveis abordados nos artigos objeto de análise de conteúdo 
ou 3) indicadores/decisão. Podemos constatar que são em maior número os artigos sobre o papel e relevância dos SIS/SIE para a gestão eficiente dos cuidados. Seguem-se, em menor número, os trabalhos que estudam os SIS/SIE numa perspetiva mais analítica, em termos de avaliação de resultados e contributo dos registos eletrónicos (melhor diagnóstico/qualidade do cuidado). E ainda em menor número estão os estudos sobre o contributo dos SIS/SIE em delinear indicadores para a tomada de decisão (estratégias de informação e desempenho em saúde). Esta tabela sugere assim uma importância crescente dos SIS/SIE como indicadores de qualidade dos cuidados prestados em saúde e enfermagem. Ressalta-se o facto de que vários dos artigos em revisão, nos três níveis indicados, referem-se a Portugal refletindo também a importância desta vertente.

\section{Análise e Discussão}

\subsection{Sistemas de Informação para a Qualidade dos Cuidados de Enfermagem}

Ao analisar os estudos inseridos nesta categoria, verificou-se que os SI são uma ferramenta de apoio à gestão e eficiência das atividades. Os SI promovem a qualidade dos cuidados, pela atualização da informação nos serviços e na gestão dos recursos. Trazem vantagens ao nível da qualidade da informação (qualidade dos registos) e dos processos (cumprimento das etapas de enfermagem). Os enfermeiros consideraram que os SIE são importantes para um bom desempenho diário. O impacto da formação especializada na aceitação dos SIE é relevante: um maior grau de formação sobre a CIPE incentiva os enfermeiros a aceitar os SI. Outro fator que favorece essa aceitação é envolver ativamente os profissionais nos processos de mudança.

O uso de SI permite aceder ao processo eletrónico do doente, elaborar diagnósticos e criar intervenções com base nos mesmos. Tais sistemas diminuem a ocorrência de erros, salvaguardando a segurança do utente de saúde. Os enfermeiros referem que as informações estão disponíveis de forma mais ampla e ágil para tomada de decisão e cuidado personalizado. Assim, os SIE promovem a personalização do atendimento e a eficácia do cuidado. Diminuem o tempo gasto a recolher informação e melhoram a comunicação entre os elementos da equipa. Assim, os SIS/SIE permitem integrar uma 'base de conhecimento' a partir dos dados registados no processo do doente. Fornecem informações em tempo real que permitem prestar o cuidado atempado.

Os registos de enfermagem constituem uma fonte de investigação e documentação legal pois permitem avaliar os cuidados prestados. Um défice de registos dificulta o exercício da profissão e a auditoria de enfermagem para avaliação da qualidade dos cuidados. Os SI têm um importante papel em reduzir as disparidades no sistema de saúde. Contudo, existem limitações à sua implementação tais como pouca familiaridade dos enfermeiros com tecnologias e com eventuais falhas nos sistemas. O sucesso de implementação dos SI depende do envolvimento ativo dos profissionais de saúde. E os enfermeiros devem primar pela qualidade dos registos que efetuam (Sousa et al., 2012).

\subsection{Sistemas de Informação como Indicador de Qualidade dos Cuidados}

A aplicação de indicadores de qualidade para avaliar a prestação de cuidados de enfermagem envolve aspetos relacionados com as atividades desenvolvidas entre 
enfermeiros e utentes. Nos estudos analisados nesta categoria, foi possível verificar que os bons registos eletrónicos contribuem para a continuidade do cuidado e segurança do utente. Podem assim funcionar como indicadores de qualidade, pela avaliação dos cuidados de forma sistemática. Nesse sentido, ajudam a priorizar casos e alertam para aspetos que podem passar despercebidos (criação de sistemas de alerta, indicação de situações críticas).

A qualidade dos cuidados pode ser avaliada por indicadores de ordem técnica, ambiental, relacional e ética. Ao nível institucional, o uso de indicadores reflete maior reconhecimento, respeito profissional, equipas melhor informadas, menor tempo de internamento, melhor gestão dos recursos materiais, aumento da satisfação do utente e família. Isso contribui para diminuir os custos da instituição e do SNS. Os indicadores são instrumentos que funcionam como uma medida que pode ser usada como guia para monitorizar a qualidade dos cuidados e serviços prestados. Sinalizam divergências e atuam como alerta para aspetos que necessitam ser mudados (Vituri \& Matsuda, 2009). A melhoria da qualidade é um processo amplo, dinâmico e requer do profissional de enfermagem conhecimentos baseados na evidência. O controlo da qualidade pode ser alicerçado nos indicadores e está relacionado com o desempenho das pessoas, satisfação do cliente e cultura organizacional. A monitorização de indicadores necessita do SIE, pois a enfermagem possui um complexo conjunto de dados relativos à sua atividade. Para avaliar bem os resultados dos cuidados de enfermagem é necessário fazer registos fidedignos que traduzam a realidade dos cuidados prestados pela equipa. É com base nisto que é fulcral padronizar um conjunto mínimo de dados para que os registos de enfermagem possuam sequência lógica, descrevam a situação clínica do utente e demonstrem as intervenções efetuadas para atender às suas necessidades reais.

\section{Conclusão}

O controlo de qualidade e desempenho constitui uma das maiores preocupações de um sistema de saúde. Os estudos analisados evidenciam a relevância dos SI como um suporte às práticas de enfermagem. Disponibilizam informações de forma fácil e segura, sendo uma ferramenta de apoio à gestão. Promovem a personalização do cuidado e repensam os cuidados prestados. Os estudos aqui comparados referem que as organizações de saúde onde existem SIE promovem a qualidade e continuidade dos cuidados (Cunha et al., 2010). A maioria dos artigos revistos evidenciam que os SIE permitem uma constante atualização do processo do utente, fomentando a personalização do cuidado, o acesso de outros profissionais e redução do risco de acessos não autorizados (Lima et al., 2011).

Atualmente os SIS constituem uma fonte indispensável de suporte à decisão e prática clínica, contribuindo para aumentar o conhecimento, segurança e eficiência do processo de saúde (Pinto, 2009; Mota, 2010; Guedes, 2011). Isso depende da qualidade com que os dados são produzidos e partilhados no SI. A avaliação da qualidade dos cuidados passa indiscutivelmente pela qualidade dos registos (Azevedo, 2010). Uma lenta ou insuficiente atualização dos SIE e a possibilidade de ficarem inoperantes contribuem para uma atitude menos favorável à sua utilização. A qualidade dos cuidados pode ser auditada através dos registos efetuados. Por isso, os enfermeiros devem primar por registos completos e detalhados, essenciais para a prática baseada na evidência (Santos \& Rennó, 2013). 
Os SIE permitem um acesso mais produtivo a indicadores de enfermagem (Labbadia et al., 2011). No entanto, a revisão feita permite constatar que ainda existe um longo caminho a percorrer pois a enfermagem tem um grande desafio que é a falta de enfermeiros plenamente envolvidos com os SIE. Outra dificuldade, apontada pelo BCG, é a falta de modelos de atuação orientados por resultados (Simões, 2015). Estas dificuldades podem estar relacionadas com alguma falta de integração ou incompatibilidade existente entre vários SI em saúde e enfermagem (Mota, 2010). Da revisão feita, destacamos assim duas importantes vertentes dos SIS/SIE: 1) permitem integrar uma 'base de conhecimento' a partir dos registos do processo do doente; e 2) têm um papel relevante na produção e atualização de indicadores de desempenho para a qualidade dos cuidados. A participação e envolvimento dos enfermeiros deve ser incentivada, pois é um dos principais grupos de atores da saúde, de forma a incutir a responsabilidade e potencial da correta utilização dos SIE.

\section{Referências}

Almeida, D. \& Fontes, C. (2013). Elaboração de telas eletrônicas em um sistema de informação hospitalar utilizando as classificações de enfermagem. Revista Eletrônica de Enfermagem, 15(4), 956-964.

Ascenção, H. (2010). Da qualidade dos cuidados de enfermagem à satisfação das necessidades do utente. Tese de Mestrado em Ciências de Enfermagem. Instituto de Ciências Biomédicas Abel Salazar, Faculdade de Medicina da Universidade do Porto.

Azevedo, P. (2010). Partilha de informação de enfermagem sobre os prestadores de cuidados: Dimensão relevante para a transição de cuidados. Tese de Mestrado em Ciências de Enfermagem. Instituto de Ciências Biomédicas Abel Salazar, Faculdade de Medicina da Universidade do Porto.

Azevedo, P. \& Sousa, P. (2012). Partilha de informação de enfermagem: Dimensões do papel de prestador de cuidados. Revista Enfermagem Referencia, III(7), 113-122.

Barra, D. \& Sasso, G. (2011). Padrões de dados, terminologias e sistemas de classificação para o cuidado em saúde e enfermagem. Revista Brasileira de Enfermagem, 64(6), 1141-1149.

Bastos, C. \& Saraiva, M. (2009). A Qualidade dos Cuidados de Enfermagem e a Norma ISO - Aplicação Empírica no Hospital Cuf Infante Santo. Disponível em http://hdl. handle.net/10174/3643 (acedido em 24 de Agosto de 2014).

Caldana, G., Gabriel, C., Rocha, F. et al. (2013). Avaliação da qualidade de cuidados de enfermagem em hospital privado. Revista Eletrônica de Enfermagem, 15(4), 915-922.

Castro, A. (2001). Revisão Sistemática e Meta-análise. Disponível em http://metodologia. org/wp-content/uploads/2010/o8/meta1.PDF (acedido em 4 de Março de 2016).

Cunha, A., Ferreira, J. \& Rodrigues, M. (2010). Atitude dos enfermeiros face ao sistema informatizado de informação em enfermagem. Revista Enfermagem Referencia, III(1), 7-16. 
D’Innocenzo, M., Adami, N. \& Cunha, I. (2006). O movimento pela qualidade nos serviços de saúde e enfermagem. Revista Brasileira de Enfermagem, 59(1), 84-88.

Freixo, J., \& Rocha, Á. (2014). Arquitetura de Informação de Suporte à Gestão da Qualidade em Unidades Hospitalares. RISTI-Revista Ibérica de Sistemas e Tecnologias de Informação, (14), 1-15.

Gomes, I. \& Caminha, I. (2014). Guia para Estudos de Revisão Sistemática: Uma Opção Metodológica para as Ciências do Movimento Humano. Disponível em http://www. seer.ufrgs.br/Movimento/article/viewfile/41542/28358 (acedido em 3 de Julho de 2015).

Guedes, A. (2011). A aceitação do registo de saúde eletrónico pelos profissionais de saúde das instituições hospitalares. Tese de Mestrado em gestão da saúde. Escola Nacional de Saúde Publica, Universidade Nova de Lisboa.

Hesbeen, W. (2001). Qualidade em enfermagem pensamento e ação na perspetiva do cuidar. Loures: Lusociencia.

Labbadia, L., D’Innocenzo, M., Fogliano, R., Silva, G., et al (2011). Sistema informatizado para gerenciamento de indicadores da assistência de enfermagem do Hospital de São Paulo. Revista da Escola de Enfermagem da USP, 45(4), 1013-1017.

Lima, A. \& Melo, T. (2011). Informatização da documentação clínica de enfermagem: Expectativas das enfermeiras de implementação. Revista Enfermagem Referencia, III(4), 81-88.

Lima, D., Braga, A., Fernandes, J. \& Brandão, E. (2011). Sistema de informação em saúde: Conceções e perspetivas dos enfermeiros sobre o prontuário eletrónico do paciente. Revista Enfermagem Referencia, III(5), 113-119.

Martins, A., Pinto, A., Lourenço, C., Pimentel, E., Fonseca, I., André, M., Almeida M., Mendes, O. \& Santos, R. (2008). Qual o lugar da escrita sensível nos registos de enfermagem?. Pensar Enfermagem, 12(2), 52-61.

Moher, D., Liberati, A., Tetzlaff, J. \& Altman, D. (2009). Preferred reporting items for systematic reviews and meta-analyses: The PRISMA statement. PLOS Medicine, 6(7), 1-6.

Mota, L. (2010). SI de enfermagem: Um estudo sobre a relevância da informação para os médicos. Tese de Mestrado de Informática Médica. Instituto de Ciências Biomédicas Abel Salazar, Faculdade de Medicina da Universidade do Porto.

Mota, L., Pereira, F. \& Sousa, P. (2014). SI de enfermagem: Exploração da informação partilhada com os médicos. Revista Enfermagem Referencia, IV(1), 85-91.

Ordem dos Enfermeiros (2007). Resumo mínimo de dados e core de indicadores de enfermagem para o repositório central de dados da saúde. Lisboa, Portugal.

Pinto, L. (2009). SI e profissionais de enfermagem. Dissertação de Mestrado em Gestão dos Serviços de Saúde. Universidade de Trás-os-Montes e Alto Douro. 
PNS - Plano Nacional de Saúde (2012-2016). Eixo Estratégico - Qualidade em Saúde. Disponível em http://pns.dgs.pt/files/2012/02/o024_-_Qualidade_em_ Sa\%C3\%BAde_2013-01-17_.pdf (acedido em 24 de Agosto de 2014).

Pocinho, M. (2008). Lições de Metanálise. Disponível em http://docentes.ismt.pt/ m pocinho/Licoes_de_revisao_sistematica_e_metanalise.pdf (acedido em 1 de Julho de 2015).

Rocha, Á., \& Rocha, B. (2014). Adopting nursing health record standards. Informatics for Health and Social Care, 39(1), 1-14. Doi: 10.3109/17538157.2013.827200

Sampaio R. \& Mancini M. (2007). Estudos de revisão sistemática: Um guia para síntese criteriosa da evidência científica. Revista Brasileira de Fisioterapia, 11(1), 83-89.

Santos, M. \& Rénno, C. (2013). Indicadores de qualidade da assistência de enfermagem em centro cirúrgico: Revisão integrativa da literatura. $R A S, 15(58), 27-36$.

Sasso, G., Barra, D., Paese, F., Rios, G., Marinho, M., \& Debétio, M. (2013). Processo de enfermagem informatizado: Metodologia para associação da avaliação clínica, diagnósticos, intervenções e resultados. Revista da Escola de Enfermagem da USP, 47(1), 242-249.

Setz, V. \& D’Innocenzo, M. (2009). Avaliação da qualidade dos registos de enfermagem no prontuário por meio de auditoria. Acta Paulista de Enfermagem, 22(3), $313-317$.

Simões, B. (2015). Portugal tem um dos melhores sistemas de saúde do mundo, mas pode perdê-lo. Disponível em http://www.jornaldenegocios.pt/economia/detalhe/ portugal_tem_um_dos_melhores_sistemas_de_saude_do_mundo_mas_pode_ perde_lo.html (acedido em 3 de Março de 2016).

Sousa, P. (2005). O sistema de partilha de informação em enfermagem entre contextos de cuidados de saúde - Um modelo explicativo. Tese de doutoramento. Instituto de Ciências Biomédicas Abel Salazar, Faculdade de Medicina da Universidade do Porto.

Sousa, P., Sasso, G. \& Barra, D. (2012). Contribuições dos registos eletrónicos para a segurança do paciente em terapia intensiva: Uma revisão integrativa. Texto \& Contexto - Enfermagem, 21(4), 971-979.

Vituri, D. \& Matsuda, L. (2009). Validação de conteúdo de indicadores de qualidade para avaliação do cuidado de enfermagem. Revista da Escola de Enfermagem da USP, 43(2), 429-437. 